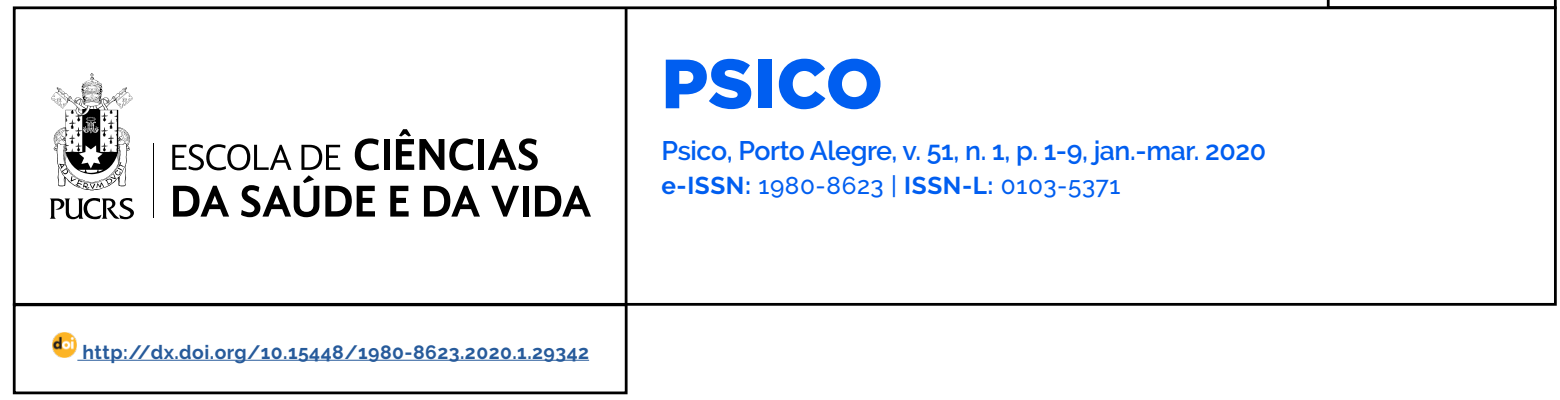

ARTIGOS

\title{
Valores sociais atenuam sintomas depressivos em vitimas de bullying
}

Social values attenuate depressive symptoms in victims of bullying

Los valores sociales atenúan los sintomas depresivos en las victimas de acoso escolar

Renan Pereira Monteiro' orcid.org/0000-0002-5745-3751 renanpmonteiro@gmail.com

\section{Emerson Diógenes de Medeiros $^{2}$}

orcid.org/0000-0002-1407-3433 emersondiogenes@gmail.com

\section{Carlos Eduardo}

Pimentel ${ }^{3}$

orcid.org/0000-0003-3894-5790

kdu1976@gmail.com

Rildésia Silva Veloso

Gouveia ${ }^{4}$

orcid.org/0000-0002-3267-7855 rsvgouveia@gmail.com

\section{Valdiney Veloso \\ Gouveia ${ }^{5}$}

orcid.org/0000-0003-2107-5848 vvgouveia@gmail.com

Recebido em: 3 dez. /2017 Aprovado em: 28 ago. 2019. Publicado em: XX XXX. 2020.
Resumo: O presente estudo objetivou conhecer em que medida sofrer bullying prediz sintomas depressivos, além de verificar o papel moderador dos valores nessa relação. Participaram 455 crianças ( $M_{\text {idade }}=11,36 ; D P=1,52 ; 53.5 \%$ meninas). Os resultados indicaram que sintomas depressivos foram preditos por vitimização de bullying, baixos escores em valores suprapessoais, normativos e interativos. Ademais, observou-se que os dois últimos moderaram a relação entre depressão e bullying, sendo essas relações mais fortes entre os infantes que dão pouca importância a valores sociais. Os resultados são discutidos à luz da literatura, sendo propostas possibilidades de intervenção pautada na promoção de valores, buscando atenuar os efeitos negativos do bullying.

Palavras-chave: depressão, bullying, vitimização, valores sociais.

Abstract: The present study aimed to know to what extent to suffer bullying predicts depressive symptoms, besides verifying the moderating role of human values in this relation. A total of 455 children $(M=11.36, S D=1.52,53.5 \%$ girls $)$ participated in this study. The results indicated that depressive symptoms were predicted by victimization of bullying, low scores in suprapersonal, normative and interactive values. Additionally, it was observed that the last two moderated the relation between depression and bullying, being these associations stronger among those participants that give little importance to social values. The results are discussed considering the literature, being suggest possibilities of intervention based on values, seeking to mitigate the negative effects of bullying.

Keywords: depression, bullying, victimization, social values.

Resumen: El presente estudio objetivó conocer en qué medida sufrir acoso escolar predice síntomas depresivos, además de verificar el papel moderador de los valores en esta relación. Participaron 455 niños $\left(M_{\text {edad }}=11,36, D E=1,52 ; 53.5 \%\right.$ niñas). Los resultados indicaron que los sintomas depresivos fueron predichos por la victimización de acoso escolar, bajas puntuaciones en valores suprapersonales, normativos y interactivos. Además, se observó que los dos últimos moderaron la relación entre depresión y acoso, siendo estas relaciones más fuertes entre los niños que dan poca importancia a valores sociales. Los resultados son discutidos a la luz de la literatura, siendo propuestas posibilidades de intervención pautada en la promoción de valores, buscando atenuar los efectos negativos del acoso. Palabras clave: depresión, acoso escolar, victimización, valores sociales. 
A escola tem um papel nuclear na vida das pessoas, contribuindo não apenas com o avanço no sistema formal de ensino, mas também sendo um dos primeiros espaços de socialização e internalização de normas. Entretanto, muitos contextos escolares não cumprem o seu papel como locais que promovem o aprendizado, tampouco o desenvolvimento emocional, social e cognitivo das crianças e adolescentes. Diversas são as variáveis que contribuem para esse quadro desfavorável, a exemplo do ambiente hostil que muitos alunos encontram na escola, onde são maltratados, sofrendo agressões de seus pares (Malta et al., 2014).

A agressão entre pares, ou bullying, é uma das principais variáveis que podem tornar o ambiente escolar insalubre. A propósito, dados têm apontado que aproximadamente $8 \%$ dos escolares brasileiros sofrem bullying (Malta et al., 2014). Esse fenômeno pode ser compreendido como ações agressivas, expressas de variadas formas (e.g., apelidar, bater e excluir de grupos), que um individuo ou grupo pratica, de forma repetida e intencional, contra um colega que se encontra em uma relação desigual de poder (Farrington, 1993).

A incidência da vitimização de bullying preocupa, sobretudo, se analisarmos as suas consequências negativas. Evidências empíricas indicam que crianças que sofrem agressões repetidamente reportam baixa autoestima, niveis maiores de medo e de risco percebido de vitimização, avaliando a escola como um lugar que não é seguro (Esbensen \& Carson, 2009), além de ter niveis menores de bem-estar (Hellfeldt, Gill, \& Johansson, 2016). Ademais, são consistentes as relações entre ser vitima de bullying e apresentar sintomas de depressão e ansiedade, além de pobre saúde geral e ideação suicida, podendo, inclusive, o jovem ser mais propenso a usar substâncias ilícitas (Bannink, Broeren, van de Looij-Jansenn, Waart, \& Raat, 2014; Perren, Dooley, Shaw, \& Cross, 2010; Moore et al., 2017).

A depressão pode tornar as vitimas de bullying mais vulneráveis ao consumo de drogas, tendo um papel mediador (Luk, Wang, \& Simons-Morton, 2011), além da depressão maior na idade adulta ser predito pelo sofrimento de bullying na infância
(Copeland, Wolke, Angold, \& Costello, 2013; Ttofi, Farrington, Lösel, \& Loeber, 2011). A propósito do anteriormente comentado, estudos longitudinais têm verificado que ser vítima de bullying na infância tem maior impacto adverso para a vida adulta do que sofrer tais agressões na adolescência, indicando a importância de prevenir esse problema nas idades escolares iniciais (Hoffman, Phillips, Daigle, \& Turner, 2016). Ainda sobre os impactos que a vitimização pode ter na saúde mental, Evans-Lacko et al. (2017) verificaram que, comparado com pessoas que não sofreram bullying na infância, aqueles que foram frequentemente agredidas foram mais propensas a fazer uso de serviços de saúde mental na infância, adolescência e na meia idade. Ademais, pessoas que sofreram bullying na adolescência apresentaram tendências para a prática de comportamentos sexuais de risco e uso de substâncias durante os anos de faculdade (Kritsotakis, Papanikolaou, Androulakis, \& Philalithis, 2017).

Portanto, a literatura é consistente ao apontar que o bullying pode parar, mas os seus efeitos podem seguir para o resto da vida, indicando a relevância do problema e a necessidade de mais atenção de pais e professores para cessar tais agressões. Além disso, é fundamental conhecer variáveis que possam representar um fator de proteção contra o envolvimento em bullying ou mesmo aquelas que podem amenizar os efeitos desastrosos que a vitimização pode acarretar. Um dos principais fatores de proteção com relação à vitimização, ou redução de seus efeitos adversos, é o apoio social. Contar com pais, outros adultos e amigos que ajudam, se preocupam e dão suporte, é um aspecto fundamental para amenizar o sofrimento de agressões, reduzindo os impactos negativos que essas possam ocasionar (Borowsky, Taliaferro, \& Morris, 2013; Stadler, Feifel, Rohrmann, Vermeiren, \& Poustka, 2010; Yeung Thompson \& Leadbeater, 2013). Ademais, Stadler et al. (2010) indicam que um clima escolar positivo, ter suporte dos professores e apego à escola também podem ser fatores de proteção, amenizando os efeitos da vitimização.

Em outro estudo, Bollmer, Milich, Harris e Maras (2005) verificaram que contar com amizades de 
alta qualidade atenuam a prática de bullying entre crianças com problemas externalizantes. Apesar de verificarem que a falta de tais amizades e problemas internalizantes estarem relacionados a ser vitima de bullying, os autores não encontraram o efeito moderador da qualidade da amizade na relação entre as duas últimas variáveis. Ainda sobre a falta de suporte, Baker e Bugay (2011) observaram que a solidão cumpre um papel mediador na relação entre vitimização e sintomas depressivos, isto é, a solidão aumenta os efeitos negativos de sofrer bullying, elevando o risco de depressão.

A partir do exposto, parece que contar com uma rede de suporte reduz as consequências negativas de sofrer bullying. De fato, ter pessoas próximas com quem se pode contar para desabafar ou enfrentar a vitimização, falando sobre as suas experiências negativas, pode ser um fator protetivo, reduzindo o sofrimento (Stadler et al., 2010). Para além do suporte recebido, é fundamental verificar a disposição que as pessoas têm para se engajar em grupos e/ou relacionamentos próximos e o papel que essa orientação social pode ter minorando as consequências da vitimização. Concretamente, estudos têm explorado o papel dos valores sociais como fatores de proteção contra o envolvimento, no papel de agressor, em ações de bullying (Knafo, Daniel, \& Khoury-Kassabri, 2008; Monteiro et al., 2017), contudo, pouco se sabe acerca do papel que esse construto pode ter nas vítimas.

De forma geral, os valores são princípios-guia gerais que transcendem situações e objetos específicos, expressando cognitivamente as necessidades e orientando comportamentos (Gouveia, Milfont, Vione, \& Santos, 2015). De acordo com a Teoria Funcionalista dos Valores Humanos (Gouveia, Milfont, \& Guerra, 2014), existem seis subfunções dos valores, descrevendo perfis que são orientados pela busca de emoções, novas e excitantes experiências (experimentação), que priorizam o reconhecimento, posições de destaque e liderança (realização), os que se pautam na busca por estabilidade e condições que garantam a sobrevivência (existência), aqueles que priorizam a busca por informação e conhecimento, reconhecendo a importância de ideias abstratas em detrimento de aspectos materiais (suprapessoal), os que se orientam por normas e regras claras, respeitando as hierarquias (normativo) e, por fim, os que dão importância a fazer parte de grupos sociais, ter amizades próximas e contar com suporte social (interativa).

Avaliando os estudos prévios que ressaltam os vínculos sociais como fatores importantes que atenuam o efeito do bullying, é plausivel pensar no importante papel que os valores da subfunção interativa podem cumprir. Estima-se que as pessoas que tenham necessidade de amor e afiliação orientam-se por valores dessa subfunção, que guiará o comportamento das pessoas no sentido de satisfazer tais necessidades (Coelho, Maio, Gouveia, Wolf, \& Monteiro, 2017; Gouveia et al., 2014). De acordo com Gouveia (2013), essa subfunção dos valores enfatiza experiências afetivas compartilhadas entre as pessoas, sendo essencial para estabelecer, regular e manter as relações interpessoais. Os valores específicos dessa subfunção ressaltam aspectos da vida social, sobretudo no compartilhamento de sentimentos, emoções e experiências (afetividade), envolvendo as relações pessoa-grupo, ressaltando um senso de identidade e pertença a um grupo social (convivência) e a ênfase em relações próximas, assegurando o suporte social e afastando o isolamento que pode acarretar em solidão (apoio social; Gouveia, 2013; Medeiros, 2011).

Ademais, associa-se a subfunção interativa a maiores niveis de bem-estar subjetivo (Nascimento, 2016) e crescimento pós-traumático (Medeiros, Couto, Fonseca, Brito, \& Castro, 2016). Medeiros et al. indicam que a priorização dos valores interativos possibilita oportunidades para que o individuo que sofreu o trauma, ou as vitimizações, desabafe repetidamente sobre a sua situação com pessoas próximas, podendo reinterpretar a situação, vendo possibilidades de superar e crescer em meio às adversidades. Nesta direção, presume-se que os valores interativos podem representar um fator de proteção amenizando os impactos do bullying nas vítimas. Especificamente, a partir da literatura consultada, no presente estudo serão testadas duas hipóteses: (1) sofrer bullying irá predizer sintomas depressivos 
e (2) essa relação será mais forte no grupo que atribui pouca importância aos valores interativos.

\section{Método}

\section{Participantes e procedimento}

Participaram 455 estudantes $\left(M_{\text {idade }}=11,36\right.$; $D P=1,52)$, em maioria meninas $(53,5 \%)$ e de escola pública (65,8\%). Para a coleta dos dados, inicialmente entrou-se em contato com os diretores das escolas, solicitando a permissão para a aplicação dos questionários. Posteriormente, por se tratar de uma amostra composta por menores, enviou-se um termo de Consentimento Livre e Esclarecido para os pais e responsáveis, solicitando a permissão para que os infantes participassem. No termo haviam informações sobre o estudo, indicando o caráter voluntário e anônimo, sendo que a pesquisa recebeu parecer favorável do comitê de ética em pesquisa da Universidade Federal do Piaui (CAAE: 0193.0.045.000-11). Ademais, os dados ora apresentados fazem parte de um projeto maior que objetivou construir medidas para avaliação do bullying (agressores e vítimas), além de verificar os seus correlatos.

\section{Instrumentos}

Os participantes receberam um livreto contendo questões demográficas (e.g., sexo, idade, tipo de escola) e as seguintes medidas:

Escala de Vitimização de Bullying (EVB). Esta medida é composta por 30 itens, questionando a frequência com que os alunos sofreram agressões (e.g., Me apelidaram; Me deram socos ou murros) em sua escola na última semana (o = Nenhuma vez; 4 = Quatro ou mais vezes).

\section{Inventário de Depressão Infantil (CDI; Kovács,}

1983). Utilizou-se a versão adaptada para o contexto brasileiro por Gouveia, Barbosa, Almeida e Gaião (1995), sendo esta medida formada por 20 itens que avaliam sintomas somáticos, afetivos, cognitivos e comportamentais da depressão. Especificamente, cada item oferece três possibilidades de respostas, indicando ausência, presença do sintoma e o seu agravo.

Questionário dos Valores Básicos Infantil (QVB-I; Gouveia, Milfont, Soares, Andrade, \&
Leite, 2011). Esta medida é composta por 18 itens ou valores especificos adaptados para crianças (e.g., Convivência. Conviver bem com familiares e vizinhos; ter amigos(as) no colégio ou no bairro; e participar de atividades e brincadeiras com os(as) amigos(as); Afetividade. Ter uma relação de afeto profunda com seus(suas) irmãos(ãs) e/ou familiares; ter alguém com quem compartilhar suas alegrias, tristezas e segredos; e ter um(a) amigo(a) intimo(a) em quem confiar). Os itens são respondidos em escala de cinco pontos ( 1 = Nenhuma importância; 5 = Máxima importância), e distribuidos em seis fatores ou subfunções valorativas.

\section{Análise de Dados}

Os dados foram analisados por meio do software IBM SPSS em sua versão 20. Foram realizadas estatísticas descritivas (caracterização da amostra), análise de correlação $r$ de Pearson (conhecer o padrão de relações entre as variáveis), além da regressão hierárquica (verificar o papel moderador dos valores na relação entre vitimização e depressão). Por fim, utilizou-se o programa ModGraph - I (Jose, 2013) para gerar as figuras da moderação e calcular os simple slopes, que indicam para quais grupos (baixo, médio e alto) a interação é estatisticamente significativa.

\section{Resultados}

Inicialmente, verificou-se que o escore total da medida de depressão se correlacionou $(p<$ 0,05 ) positivamente com o sofrimento de bullying e negativamente com valores das subfunções existência, suprapessoal, normativa e interativa (Tabela 1). Especificamente, uma análise de regressão (método Stepwise) indicou que os preditores dos sintomas depressivos foram o sofrimento de bullying $(B=0,13 ; \mathrm{IC} 95 \%=0,08 / 0,19 ; \beta$ $=0,30 ; t=5,35 ; p<0,001$ ) e os valores das subfunções suprapessoal $(B=0,06 ; \mathrm{IC} 95 \%=0,02 / 0,10 ; \beta=0,21 ; t$ $=3,16 ; p<0,01)$, normativa $(\mathrm{B}=-0,07$; IC $95 \%=-0,11 /$ $0,03 ; \beta=-0,25 ; t=-3,56 ; p<0,001)$ e interativa $(B=$ $-0,08 ;$ IC $95 \%=-0,12 /-0,03 ; \beta=-0,21 ; t=-3,06 ; p<$ 0,01 ). O modelo de regressão com essas quatro variáveis explicou $24 \%$ da variabilidade nos escores da medida de depressão. 
TABELA 1 - Média, Desvio Padrão e Correlação entre depressão, vitimização e valores

\begin{tabular}{|c|c|c|c|c|c|c|c|c|c|}
\hline & $M$ & $D P$ & & & & & & & \\
\hline 1 & 1,29 & 0,24 & & & & & & & \\
\hline 2 & 0,54 & 0,51 & $0,34^{* *}$ & & & & & & \\
\hline 3 & 4,00 & 0,86 & $-0,02$ & $0,10^{*}$ & & & & & \\
\hline 4 & 2,96 & 0,99 & $-0,04$ & $0,18^{* *}$ & $0,46^{* *}$ & & & & \\
\hline 5 & 4,48 & 0,69 & $-0,16^{* *}$ & $-0,06$ & $0,43^{* *}$ & $0,25^{* *}$ & & & \\
\hline 6 & 3.93 & 0,85 & $-0,09^{*}$ & 0,03 & $0,55^{\star *}$ & $0,30^{* *}$ & $0,44^{* *}$ & & \\
\hline 7 & 4,21 & 0,80 & $-0,24^{* *}$ & $-0,06$ & $0,29^{* *}$ & $0,12^{* *}$ & $0,38^{* *}$ & $0,46^{* *}$ & \\
\hline \multirow[t]{2}{*}{8} & 4,36 & 0,74 & $-0,19^{* *}$ & $-0,08$ & $0,44^{* *}$ & $0,16^{* *}$ & $0,50^{* *}$ & $0,42^{* *}$ & $0,54^{* *}$ \\
\hline & & & 1 & 2 & 3 & 4 & 5 & 6 & 7 \\
\hline
\end{tabular}

Nota: ${ }^{*} p<0,05,{ }^{* *} p<0,001$ (teste uni-caudal). Identificação das variáveis: $1=$ Depressão, $2=$ Vitimização, 3 = Experimentação, 4 = Realização, 5 = Existência, 6 = Suprapessoal, 7 = Normativa e 8 = Interativa.

A partir do padrão de associações entre as variáveis, o passo seguinte foi testar o papel moderador dos valores humanos (subfunções suprapessoal, normativa e interativa), na relação entre o sofrimento de bullying e a presença de sintomas depressivos. Três análises de regressão hierárquica foram realizadas, uma para cada subfunção valorativa. No primeiro passo de todas as análises entraram-se com os escores padronizados das subfunções e da medida de vitimização, e no segundo passo com o termo de interação (Tabela 2).

TABELA 2 - Regressões hierárquicas

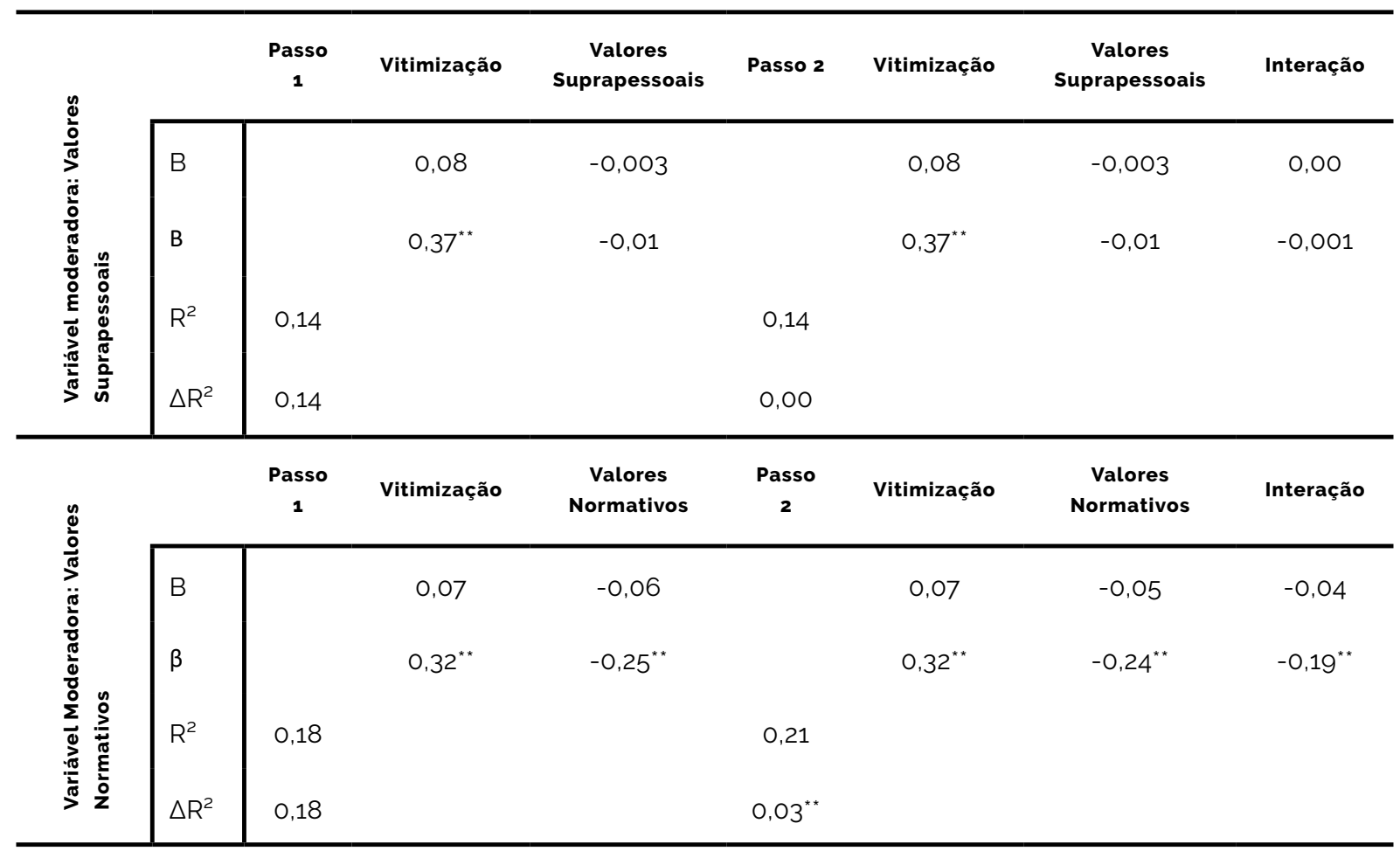




\section{$6 / 9$}

Psico, Porto Alegre, v. 51, n. 1, p. 1-9, jan.-mar. 2020 | e-29342

\begin{tabular}{|c|c|c|c|c|c|c|c|c|}
\hline & & $\begin{array}{c}\text { Passo } \\
1\end{array}$ & Vitimização & $\begin{array}{c}\text { Valores } \\
\text { Interativos }\end{array}$ & Passo 2 & Vitimização & $\begin{array}{c}\text { Valores } \\
\text { Interativos }\end{array}$ & Interação \\
\hline 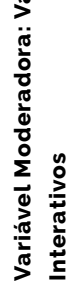 & $\begin{array}{l}B \\
\beta \\
R \\
\Delta R^{2}\end{array}$ & $\begin{array}{l}0,18 \\
0,18\end{array}$ & $\begin{array}{c}0,07 \\
0,32^{* *}\end{array}$ & $\begin{array}{l}-0,06 \\
-0,24^{* *}\end{array}$ & $\begin{array}{c}0,20 \\
0,02^{* *}\end{array}$ & $\begin{array}{c}0,07 \\
0,33^{* *}\end{array}$ & $\begin{array}{l}-0,05 \\
-0,21^{* *}\end{array}$ & $\begin{array}{l}-0,03 \\
-0,14^{* *}\end{array}$ \\
\hline
\end{tabular}

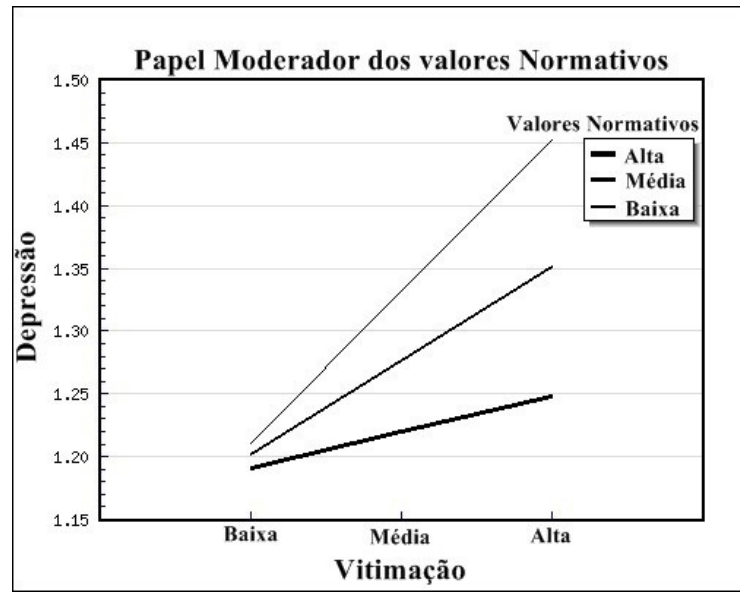

Figure 1 - Papel moderador dos valores sociais

De acordo com a Tabela 2, os valores suprapessoais não predisseram a depressão, ademais, o termo de interação (zSuprapessoal $x$ zVitimização) também não teve papel preditivo, descartando uma eventual moderação. Por outro lado, os valores normativos predisseram a depressão, assim como o termo de interação o fez, indicando moderação. Por meio do ModGraph - I (Jose, 2013), observou-se que as relações positivas entre vitimização e depressão são mais fortes entre os infantes que dão pouca (simple slope $=0,12 ; \mathrm{t}=6,77 ; \mathrm{p}<0,001$ ) ou média importância (simple slope = 0,07; $t=6,15 ; p<0,001$ ) a valores normativos (Figura 1). Não obstante, não se verificou essa interação para os elevados niveis de valores normativos (simple slope $=0,03 ; \mathrm{t}=1,61$; $p=0,11$. Por fim, os valores interativos também foram preditores da depressão, assim como o termo de interação (zInterativa $\times$ zVitimização), confirmando-se, portanto, o papel moderador dessa subfunção. O Mod-Graph-I (Jose, 2013) foi novamente utilizado para gerar a figura da

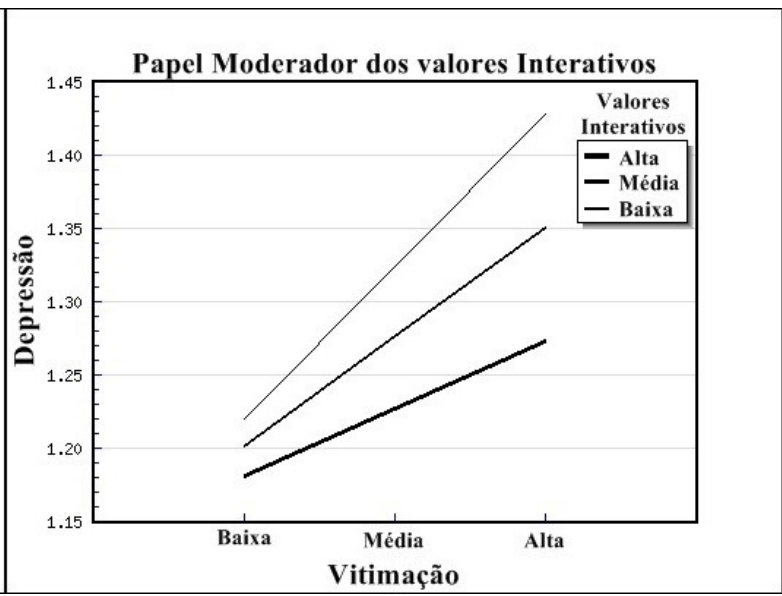

moderação (Figura 1). É possivel observar que as relações positivas entre vitimização e depressão são mais fortes entre os infantes que fazem parte dos grupos com niveis baixos (simple slope $=0,10$; $\mathrm{t}=6,12 ; \mathrm{p}<0,001)$ e intermediários de valores interativos (simple slope $=0,07 ; \mathrm{t}=5,87 ; \mathrm{p}<0,001$ ). As relações entre sofrer bullying e depressão são mais fracas no grupo que prioriza em maior medida valores da subfunção interativa (simple slope $=0,04 ; t=2,77 ; p<0,001$.

\section{Discussão}

Tendo em vista os efeitos nefastos da vitimização do bullying (Moore et al., 2017; Perren et al., 2010), é fundamental buscar eventuais fatores de proteção que possam minorar tais consequências negativas. Com esse propósito o presente estudo foi desenvolvido, verificando o papel que os valores têm na relação entre sofrer agressões dos pares e desenvolver sintomas depressivos, um dos mais frequentes correlatos 
associado às vítimas de bullying

Observou-se que sofrer bullying prediz sintomas depressivos, confirmando a Hipótese 1. Os achados deste estudo vão na mesma direção daqueles encontrados na literatura, apontando que a vitimização está relacionada com problemas de internalização, entre eles a depressão (Perren et al., 2010; Reijntjes, Kamphuis, Prinzie, \& Telch, 2010; Ttofi, Farrington, Lösel, \& Loeber, 2011). Essas relações são consistentes e o presente estudo aporta com novas evidências sobre tais relações entre escolares brasileiros.

Para além de verificar o padrão de relações supracitado, o presente estudo reuniu evidências que demonstram a utilidade dos valores para atenuar as consequências negativas da vitimização, confirmando a Hipótese 2. Neste contexto, os valores sociais (normativo e interativo) se configuram como importantes fatores de proteção, pois descrevem um perfil que valoriza experiências afetivas, o estabelecimento e manutenção de relações interpessoais, tendo, consequentemente uma rede de suporte mais extensa. Especificamente, pessoas pautadas por valores interativos compartilham com as demais sentimentos, emoções e experiências.

Ter uma rede de apoio que se possa contar para desabafar e enfrentar as experiências negativas (sofrer bullying), pode reduzir o sofrimento (Stadler et al., 2010), fazendo com que a vitima reinterprete a situação, visualizando possibilidades de crescer em meio às dificuldades (Medeiros et al., 2017). Ademais, o endosso a valores sociais, especificamente os interativos, asseguram o suporte social, afastando o isolamento e a solidão (Gouveia, 2013; Medeiros, 2011), que são fatores importantes e que mediam a relação entre vitimização e depressão (Baker \& Bugay, 2011). Logo, receber suporte de pais, colegas e da escola pode ser fundamental para o enfrentamento das agressões, inclusive reduzindo os seus efeitos desastrosos.

Os resultados ora apresentados mostram uma potencial aplicabilidade dos valores no contexto em questão. Concretamente, visto a magnitude da agressão entre pares (Malta et al., 2014; Medeiros et al., 2015; Monteiro et al., 2017), é pertinente pensar em propostas interventivas nas escolas, pautado na promoção de valores, efetuando palestras e campanhas que ressaltam a importância de seguir normas e respeitar os demais, assim como destacando a convivência e o estabelecimento de laços afetivos com outras pessoas. Trabalhando nessa direção seria possivel reduzir o comportamento agressivo (Monteiro et al., 2017), bem como fomentar tais valores nas vitimas, fazendo com que elas encarem a situação problema, busquem fortalecer a sua rede de apoio social, reduzindo os efeitos negativos da vitimização.

Apesar dos resultados promissores e da eventual aplicabilidade, os achados do presente estudo devem ser interpretados com cautela. Aspectos como a amostragem (de conveniência), desejabilidade social e o não controle de variáveis intervenientes (e.g., traços de personalidade) são limitações do estudo e precisam ser tidas em conta em possibilidades futuras. Ademais, estudos futuros podem ser realizados com o intuito de conhecer o papel dos valores sociais como moderadores das relações entre vitimização e outros desfechos (e.g., ansiedade, ideação suicida). Outra possibilidade seria verificar os valores dos pais como moderadores, sendo que os pais que se pautam em valores sociais poderiam ter um vínculo mais próximo com seus filhos, conversando sobre os problemas enfrentados e as eventuais agressões sofridas na escola.

Portanto, ainda há muito a ser feito. Apesar da gravidade do problema no Brasil, verifica-se uma relativa escassez de estudos que buscam conhecer variáveis preditoras do envolvimento em bullying. Por fim, o presente estudo configura-se como uma evidência em torno do importante papel das relações interpessoais, do estabelecimento de vínculos próximos e, em consequência, ter amizades de qualidade. sendo fatores protetivos contra o envolvimento em situações de bullying, além de reduzir seus efeitos (Baker \& Bugay, 2011; Bollmer et al., 2005; Stadler et al., 2010). 


\section{Referências}

Baker, Ö. E., \& Bugay, A. (2011). Mediator and moderator role of loneliness in the relationship between peer victimization and depressive symptoms. Journal of Psychologists and Counsellors in Schools, 21, 175185. https://doi.org/10.1375/ajgc.21.2.175

Bannink, R., Broeren, S., van de Looij-Jansen, P. M., de Waart, F. G., \& Raat, H. (2014). Cyber and traditional bullying victimization as a risk factor for mental health problems and suicidal ideation in adolescents. PloS one, 9, e94026. https://doi.org/10.1371/journal. pone. 0094026

Bollmer, J. M., Milich, R., Harris, M. J., \& Maras, M. A. (2005). A friend in need: The role of friendship quality as a protective factor in peer victimization and bullying. Journal of interpersonal violence, 20(6), 701712. https://doi.org/10.1177/0886260504272897

Borowsky, I. W., Taliaferro, L. A., \& McMorris, B. J. (2013). Suicidal thinking and behavior among youth involved in verbal and social bullying: Risk and protective factors. Journal of Adolescent Health, 53(1), S4-S12. https://doi.org/10.1016/j.jadohealth.2012.10.280

Coelho, G. L. H., Maio, G. R., Gouveia, V. V., Wolf, L. J., \& Monteiro, R. P. (2017). Questionário de Necessidade de Emoções (NAQ-S): Validade de construto, invariância e fidedignidade. Psico-USF, 22, 461-472. https:// doi.org/10.1590/1413-82712017220307

Copeland, W. E., Wolke, D., Angold, A., \& Costello, E. J. (2013). Adult psychiatric outcomes of bullying and being bullied by peers in childhood and adolescence. JAMA Psychiatry, 70, 419-426. https://doi. org/10.1001/jamapsychiatry.2013.504

Esbensen, F. A., \& Carson, D. C. (2009). Consequences of being bullied: Results from a longitudinal assessment of bullying victimization in a multisite sample of American students. Youth \& Society, 41(2), 209-233. https://doi.org/10.1177/0044118X09351067

Evans-Lacko, S., Takizawa, R., Brimblecombe, N., King, D., Knapp, M., Maughan, B., \& Arseneault, L. (2017). Childhood bullying victimization is associated with use of mental health services over five decades: a longitudinal nationally representative cohort study. Psychological medicine, 47, 127-135. https://doi. org/10.1017/So033291716001719

Farrington, D. P. (1993). Understanding and preventing bullying. In M. Tommy \& N. Morris, Crime and Justice (pp. 381-458). Chicago, IL: University of Chicago Press. https://doi.org/10.1086/449217

Gouveia, V. V. (2013). Teoria funcionalista dos valores humanos: Fundamentos, aplicações e perspectivas. São Paulo: Casa do Psicólogo. (DOI INEXISTENTE)

Gouveia,V., Barbosa, G., Almeida, H., \& Gaião, A. (1995). Inventário de Depressão Infantil - CDI: Estudo de adaptação com escolares de João Pessoa. Jornal Brasileiro de Psiquiatria, 44, 345-349. (DOI INEXISTENTE)
Gouveia, V. V., Milfont, T. L., \& Guerra, V. M. (2014). Functional theory of human values: Testing its content and structure hypotheses. Personality and Individual Differences, 60, 41-47. https://doi.org/10.1016/j. paid.2013.12.012

Gouveia, V. V., Milfont, T. L., Soares, A. K. S., Andrade P. R., \& Leite, I. L. (2011). Conhecendo os valores na infância: Evidências psicométricas de uma medida. PSICO, 42, 106-115. (DOI INEXISTENTE)

Gouveia, V. V., Vione, K. C., Milfont, T. L., \& Santos, W. S. (2015). Guiding actions and expressing needs: On the psychological functions of values. Psykhe, 24. 1-14. https://doi.org/10.7764/psykhe.24.2.884

Hellfeldt, K., Gill, P. E., \& Johansson, B. (2016). Longitudinal analysis of links between bullying victimization and psychosomatic maladjustment in Swedish schoolchildren. Journal of School Violence, 17(1), 1-13. https://doi.org/10.1080/15388220.2016.1222498

Hoffman, C. Y., Phillips, M. D., Daigle, L. E., \& Turner, M. G. (2016). Adult consequences of bully victimization: Are children or adolescents more vulnerable to the victimization experience?. Youth Violence and Juvenile Justice, 15, 441-464. https://doi. org/10.1177/1541204016650004

Jose, P.E. (2013). ModGraph-l: A programme to compute cell means for the graphical display of moderational analyses: The internet version, Version 3.0. Wellington, New Zealand: Victoria University of Wellington. Recuperado de https://psychology.victoria.ac.nz/ modgraph/ (DOI INEXISTENTE)

Knafo, A., Daniel, E., \& Khoury-Kassabri, M. (2008). Values as protective factors against violent behavior in Jewish and Arab high schools in Israel. Child development, 79, 652-667. https://doi.org/10.1111/j. 1467-8624.2008.01149.x

Kovacs, M. (1983). The Children's Depression Inventory: A self-rated depression scale for school age youngsters. Pittsburg, PA: University of Pittsburgh, School of Medicine. (DOI INEXISTENTE)

Kritsotakis, G., Papanikolaou, M., Androulakis, E., \& Philalithis, A. E. (2017). Associations of Bullying and Cyberbullying With Substance Use and Sexual Risk Taking in Young Adults. Journal of Nursing Scholarship, 49, 1-11. https://doi.org/10.1111/jnu.12299

Luk, J., Wang, J., \& Simons-Morton, B. (2011). Bullying victimization and substance use among US adolescents: Mediation by depression. Prevention Science, 11, 355-359. https://doi.org/10.1007/s11121-010-0179-0

Malta, D. C., Prado, R. R. D., Dias, A. J. R., Mello, F. C. M., Silva, M. A. I., Costa, M. R. D., \& Caiaffa, W. T. (2014). Bullying e fatores associados em adolescentes brasileiros: análise da Pesquisa Nacional de Saúde do Escolar (PeNSE 2012). Revista Brasileira de Epidemiologia, SUPPL, 131-145. https://doi.org/10.1590/18094503201400050011

Medeiros, E. D. D. (2011). Teoria funcionalista dos valores humanos: Testando sua adequação intra e interculturalmente (Tese de doutorado). Departamento de Psicologia, Universidade Federal da Paraiba, PB. (DOI INEXISTENTE) 
Medeiros, E. D., Couto, R. N., Fonsêca, P. N., Brito, R. C. S., \& Castro, L. S. (2017). Correlatos valorativos do crescimento pós-traumático em uma amostra brasileira. Psicologia e Saber Social, 5, 112-125. https://doi. org/10.12957/psi.saber.soc.2016.21602

Medeiros, E. D., Gouveia, V. V., Monteiro, R. P., Silva, P. G. N., Lopes, B. J., Medeiros, P. C. B., \& Silva, E. S. (2015). Escala de Comportamentos de Bullying (ECB): Elaboração e evidências psicométricas. Psico-USF, 20, 385-397. https://doi.org/10.1590/141382712015200302

Monteiro, R. P., Medeiros, E. D. D., Pimentel, C. E., Soares, A. K. S., Medeiros, H. A. D., \& Gouveia, V. V. (2017) Valores humanos e bullying: idade e sexo moderam essa relação?. Temas em Psicologia, 25, 1317-1328. https://doi.org/10.9788/TP2017.3-18Pt

Moore, S. E., Norman, R. E., Suetani, S., Thomas, H. J., Sly, P. D., \& Scott, J. G. (2017). Consequences of bullying victimization in childhood and adolescence: a systematic review and meta-analysis. World Journal of Psychiatry, 7, 60-76. https://doi.org/10.5498/w.jp.v7.i1. 60

Nascimento, A. M. (2016). Satisfação com a vida: Uma explicação pautada na crença no mundo justo e nos valores humanos (Dissertação de Mestrado). Departamento de Psicologia, Universidade Federal da Paraiba, PB. (DOI INEXISTENTE)

Perren, S., Dooley, J., Shaw, T., \& Cross, D. (2010). Bullying in school and cyberspace: Associations with depressive symptoms in Swiss and Australian adolescents. Child and Adolescent Psychiatry and Mental Health, 4, 1-10. https://doi.org/10.1186/1753-2000-4-28

Reijntjes, A., Kamphuis, J. H., Prinzie, P., \& Telch, M. J. (2010). Peer victimization and internalizing problems in children: A meta-analysis of longitudinal studies. Child abuse \& neglect, 34(4), 244-252. https://doi. org/10.1016/j.chiabu.2009.07.009

Stadler, C., Feifel, J., Rohrmann, S., Vermeiren, R., \& Poustka, F. (2010). Peer-victimization and mental health problems in adolescents: are parental and school support protective?. Child Psychiatry \& Human Development, 41, 371-386. https://doi.org/10.1007/ s10578-010-0174-5

Ttofi, M. M., Farrington, D. P., Lösel, F., \& Loeber R. (2011). Do the victims of school bullies tend to become depressed later in life? A systematic review and meta-analysis of longitudinal studies. Journal of Aggression, Conflict and Peace Research, 3, 63-73. https://doi.org/10.1108/17596591111132873

Yeung Thompson, R. S., \& Leadbeater, B. J. (2013). Peer victimization and internalizing symptoms from adolescence into young adulthood: Building strength through emotional support. Journal of Research on Adolescence, 23, 290-303. https://doi.org/10.1111/j. 1532-7795.2012.00827.x

\section{Endereço para correspondência:}

Avenida Rui Barbosa, № 473, Mato Grosso, 78075202; (86) 995417107; renanpmonteiro@gmail.com.

\section{Autor I}

Nome: Renan Pereira Monteiro.

Titulação Acadêmica: Doutor em Psicologia Social.

Afiliação Institucional: Universidade Federal de Mato Grosso (UFMT).

\section{Autor II}

Nome: Emerson Diógenes de Medeiros.

Titulação Acadêmica: Doutor em Psicologia Social.

Afiliação Institucional: Universidade Federal do Piaui (UFPI).

\section{Autor III}

Nome: Carlos Eduardo Pimentel.

Titulação Acadêmica: Doutor em Psicologia Social, do Trabalho e das Organizações.

Afiliação Institucional: Universidade Federal da Paraiba (UFPB).

\section{Autor IV}

Nome: Rildésia Silva Veloso Gouveia.

Titulação Acadêmica: Doutora em Psicologia Social.

Afiliação Institucional: Centro Universitário de João Pessoa (UNIPÊ).

\section{Autor V}

Nome: Valdiney Veloso Gouveia.

Titulação Acadêmica: Doutor em Psicologia Social.

Afiliação Institucional: Universidade Federal da Paraiba (UFPB) 